\title{
Spectral Estimation of Skin Color with Foundation Makeup
}

\author{
M. Doi ${ }^{1}$, R. Ohtsuki ${ }^{2}$, and S. Tominaga ${ }^{3}$ \\ ${ }^{1}$ Department of Telecommunications and Computer Networks, \\ Faculty of Information and Communication Engineering, \\ Osaka Electro-communication University, \\ 18-8 Hatsucho, Neyagawa, Osaka, 572-8530, Japan \\ ${ }^{2}$ Graduate School of Engineering, Osaka Electro-communication University, \\ 18-8 Hatsucho, Neyagawa, Osaka, 572-8530, Japan \\ ${ }^{3}$ Department of Engineering Informatics, \\ Faculty of Information and Communication Engineering, \\ Osaka Electro-Communication University, \\ 18-8 Hatsucho, Neyagawa, \\ Osaka, 572-8530, Japan
}

\begin{abstract}
The analysis of skin color with makeup is needed to examine the effect of makeup to human skin. Foundation is cosmetics to cover undesirable color on skin and gives basic color to skin. It is important evaluate a change of skin color by the foundation. The present paper modeled the optics of skin with foundation makeup by two layers. Then, the surface spectrum of skin with foundation makeup is estimated by the Kubelka-Munk theory for radiation transfer in the turbid medium. The proposed algorithm can predict the spectral shape of skin surface with different types of foundation by appropriately determining model parameters. As an application of the skin reflectance estimation, we have developed a color simulator for human skin with foundation makeup. Experimental results showed a good accuracy of our estimation results.
\end{abstract}

\section{Introduction}

The estimation of human skin color is important for many fields including computer graphics, medical imaging, and cosmetic development. Especially, the analysis of skin color with makeup is needed to examine the effect of makeup to human skin. Foundation is cosmetics to cover undesirable color on skin and gives basic color to the skin. Therefore it is important to evaluate a change of skin color by the foundation. There are some reports on the analysis of skin color $[1,2,5]$. In the previous paper [2], the authors described a method for estimating surface-spectral reflectance of human skin based on a skin optics model. However, there is no scientific discussion on the spectral analysis of human skin with makeup.

The present paper analyzes the surface-spectral reflectance function of human skin with foundation makeup. The main idea is based on the fact that skin color with foundation makeup can be computed from the reflectance of the skin without makeup and 
the optical property of the foundation. We propose a method for modeling human skin coloring with the makeup and estimating the surface-spectral reflectances by using the Kubelka-Munk theory [3,4]. Moreover we develop a skin color simulator based on the proposed spectral estimation method. Some essential points of the present paper are follows:

1. We do not estimate a three-dimensional color model for the human skin color, but do estimate the spectral reflectance function for the human skin surface. Note that the color model based on RGB data is device-dependent, that is unavailable for the detailed spectral analysis of skin coloring.

2. We use the Kubelka-Munk theory for calculating the transmission and reflection within the foundation layers. The Kubelka-Munk theory is used for modeling the radiation passing through a turbid medium. In this paper, the theory is applied to modeling transmission and reflection within foundation so that the surface-spectral reflectance can be predicted.

3 . We evaluate the performance of the estimation method in an experiment in detail. The accuracy of the spectral reflectances estimated for facial skin is compared with the direct measurement results using a spectrophotometer.

4. We develop a color simulator of human skin with foundation makeup by using a computer graphics technique. The skin color is created based on spectral computation using the estimated spectral reactance of a subject. The system has a graphical user interface (GUI) and displays the skin color on a calibrated CRT monitor. This system simulates the change of skin color easily in the display.

\section{Optical Model of Human Skin and Foundation}

\subsection{Human Skin}

The spectral reflectance of human skin has special features on its spectral shape. Figure 1 shows surface-spectral reflectances measured from the skin surfaces of cheek of three women by a spectrophotometer. All the spectral reflectance data are represented in the visible wavelength range from $400 \mathrm{~nm}$ to $700 \mathrm{~nm}$ through this paper. One feature is that the reflectance curves increase almost monotonically as wavelength increases. This leads to the skin object colors of pink, yellow, and brown. Another feature is the "W" shaped or "U" shaped hollow in the range from $520 \mathrm{~nm}$ to $600 \mathrm{~nm}$. This decrease in reflectance is caused by the absorption of hemoglobin. Thus, the spectral reflectance of skin is based on the influence by various pigments inside the skin tissue.

Human skin has complex optical characteristics. The skin consists of three layers of stratum corneum, epidermis and dermis. Therefore, pigments in these tissue layers influence the skin color. The epidermis has brownish pigment of melanin. The dermis has reddish pigment of hemoglobin and yellowish pigments of carotene and bilirubin. The melanin and hemoglobin cause skin color mainly. The stratum corneum does not much contribute the coloring. 


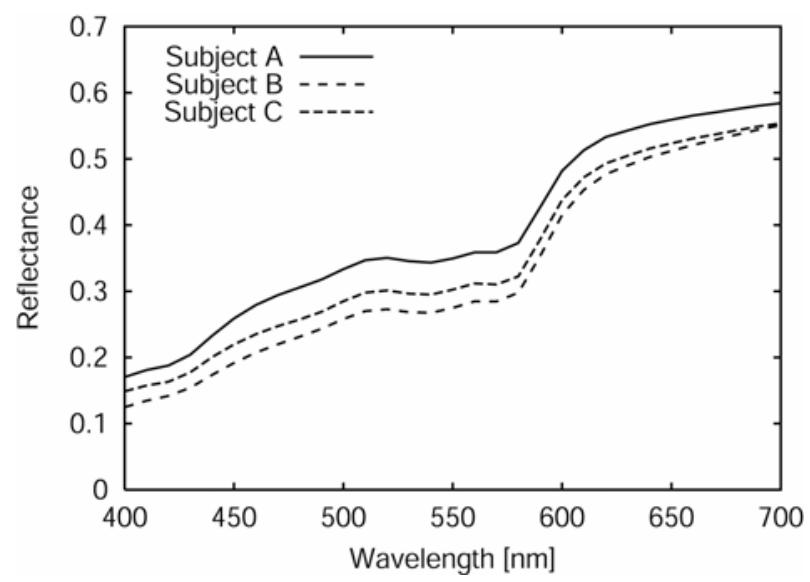

Fig. 1. Example of spectral reflectances measured from human skin

\subsection{Foundation}

In this paper, we analyze the effect of liquid foundation. The liquid foundation consists of color pigments and medium. This foundation can be regarded as a turbid material. Therefore we use the Kubelka-Munk theory for inferring skin color with makeup of the liquid foundation.

Figure 2 shows the measured spectral reflectances for three foundation materials which layer is thick enough to be opaque optically. The measurement was done with a spectrophotometer on a black background. The colors of the foundations have resemblance to the color of the skin. However, The reflectance curves do not show such "W" shaped or "U" shaped hollow as shown in skin reflectance. The skin spectra with foundation makeup depend on the depth of foundation layer. Therefore the depth is a variable parameter in our analysis.

\subsection{Optical Model}

In order to determine a relationship between the spectral reflectance function of skin with makeup and the optical characteristics for skin and foundation, an optics model is assumed for the skin with foundation makeup as shown in Figure 3. The skin optics model consists of two layers of foundation and skin, where the foundation layer is the outer layer contacting the air and the skin layer is located under the foundation layer.

It should be noted that such optical properties as scattering, refracting, and absorption in each layer are functions of wavelength. In this model, incident light is partly reflected at an interface between the foundation surface and the air. The light penetrating the interface is absorbed and scattered in the foundation layer. The light ray that reaches the skin surface is reflected and absorbed in skin layer. 


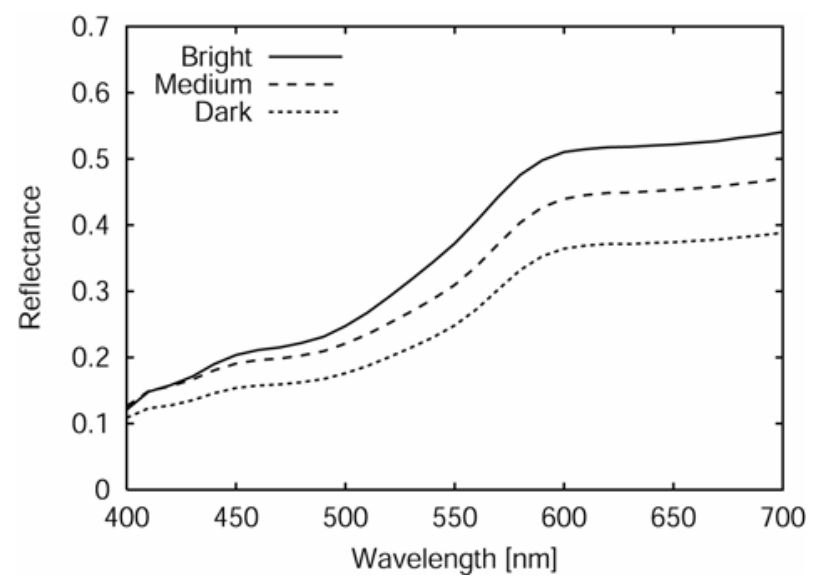

Fig. 2. Example of spectral reflectances measured from foundation materials

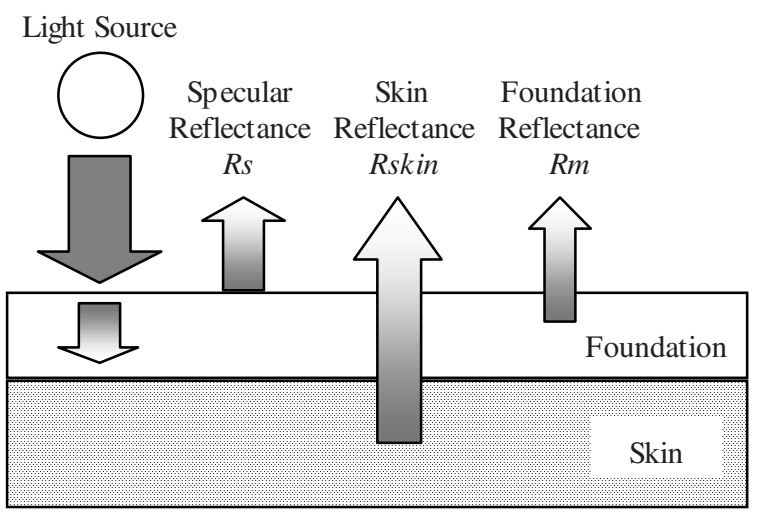

Fig. 3. Optics model for the skin with foundation makeup

\section{Kubelka-Munk Theory}

The theory by Kubelka-Munk [3,4] is used for estimating the spectral reflectance of skin with foundation makeup based on the above optics model. In general, the optical values of reflectance and transmittance within a layer consisting of turbid materials can be calculated using the Kubelka-Munk theory, where we are not consider the complex path of scattered light inside the medium. Because we assume the skin layers consisting turbid materials, use of the Kubelka-Munk theory is reasonable for the surface reflectance estimation.

Figure 4 shows the Kubelka-Munk model for radiation transfer in an absorbing medium of turbid materials. Let define the symbol $I$ be the intensity of light traveling forward direction and the symbol $J$ be the intensity of light traveling backward direction for the incident light. A relationship between the light intensities $I$ and $J$ is described as two differential equations in a variable of depth $x$ as 


$$
\begin{aligned}
& \frac{d I}{d x}=-S I-K I+S J \\
& -\frac{d J}{d x}=-S J-K J+S I
\end{aligned}
$$

where $S$ and $K$ are, respectively, coefficients of back-scattering and absorption in the media. We can derive the reflectance $R$ and the transmittance $T$ of the turbid layer with thickness $D$ from solving the above equations under some assumptions as follows

$$
\begin{aligned}
R & =\frac{1}{a+b \operatorname{coth} b S D} \\
T & =\frac{b}{a \sinh b S D+b \cosh b S D} \\
a & =\frac{S+K}{S}, b=\sqrt{a^{2}-1}
\end{aligned}
$$

When the object consists of two layers, multiple reflections in the interface between the higher layer and the lower layer is considered as shown in Figure 5. In the two-layer model, the total reflectance $R_{1,2}$ including the inter-reflection is described as

$$
R_{1,2}=R_{1}+T_{1}^{2} R_{2}\left(1+R_{1} R_{2}+R_{1}^{2} R_{2}^{2}+\cdots\right)=R_{1}+\frac{T_{1}^{2} R_{2}}{1-R_{1} R_{2}}
$$

where $T_{1}$ and $R_{1}$ are the transmittance and reflectance of Layer 1, respectively, and $R_{2}$ is the reflectance of Layer 2 .

The scattering coefficients $S$ and absorption coefficients $K$ of the layer of turbid material can be derived from the measured spectral reflectance of a thick layer of the material and thin layer of material with background by the following equations. The thick layer must be thick enough to be opaque optically.

$$
\begin{aligned}
& S=\frac{1}{b D}\left(\operatorname{coth}^{-1} \frac{a-R_{0}}{b}-\operatorname{coth}^{-1} \frac{a-R_{g}}{b}\right) \\
& K=S(a-1) \\
& \quad a=\frac{1}{2}\left(\frac{1}{R_{\infty}}+R_{\infty}\right), \quad b=\left(a^{2}-1\right)^{\frac{1}{2}}
\end{aligned}
$$

where $D$ is the thickness of the thin layer, $R_{0}$ is the reflectance of the thin layer, $R_{g}$ is the reflectance of the background of the thin layer and $R_{\infty}$ is the reflectance of the thick layer. 


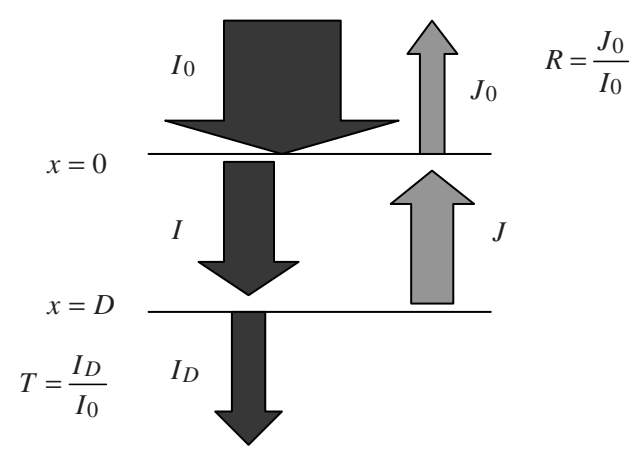

Fig. 4. The Kubelka-Munk model for a single layer

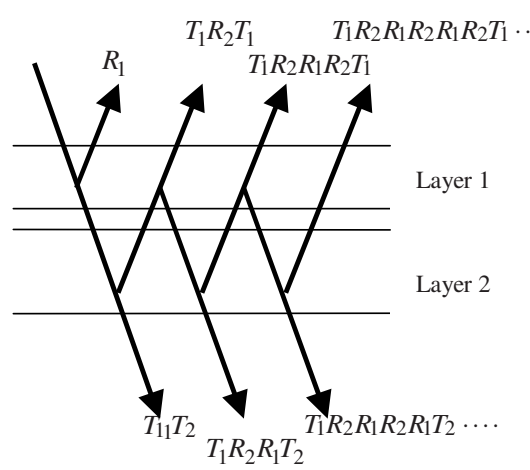

Fig. 5. Two layered model

\section{Color Estimation}

The spectrum of skin with makeup is estimated by the Kubelka-Munk theory. The object color of human skin is created from three kinds of spectral data of surfacespectral reflectance, an illuminant spectrum, and a spectral response of a display device used for computer graphics. The proposed algorithm can predict the spectral shape of skin surface by appropriately determining model parameters. We control the specular reflectance and the depth of the foundation layer.

First, we defined a set of equations for estimating spectral skin reflectance using the Kubelka-Munk theory as Equation 1.

$$
R(\lambda)=\left(1-R_{s}\right)\left(R_{m}(\lambda)+\frac{T_{m}(\lambda)^{2} R_{\text {skin }}(\lambda)}{1-R_{m}(\lambda) R_{\text {skin }}(\lambda)}\right)+R_{s}
$$

The functions of $\lambda$ in this equation are described 


$$
\begin{aligned}
& R_{m}(\lambda)=\frac{1}{a_{m}(\lambda)+b_{m}(\lambda) \operatorname{coth} D_{m} b_{m}(\lambda) S_{m}(\lambda)} \\
& T_{m}(\lambda)=\frac{b_{m}(\lambda)}{a_{m}(\lambda) \sinh D_{m} b_{m}(\lambda) S_{m}(\lambda)+b_{m}(\lambda) \cosh D_{m} b_{m}(\lambda) S_{m}(\lambda)} \\
& a_{m}=\frac{S_{m}(\lambda)+K_{m}(\lambda)}{S_{m}(\lambda)} \\
& b_{m}(\lambda)=\sqrt{a_{m}(\lambda)^{2}-1}
\end{aligned}
$$

We note that the spectral reflectance $R(\lambda)$ is determined by the two parameters; the specular reflectance between the air and the skin surface $R_{s}$ and the thickness of foundation layer $D_{m}$. We assume that $R_{s}$ doesn't depend on wavelength. $R_{\text {skin }}(\lambda)$ is the spectral reflectance of human skin. $K_{m}(\lambda)$ is the absorption of foundation and $S_{m}(\lambda)$ is the scattering of foundation. It should be noted these coefficients depend on wavelength.

The skin color with foundation makeup in RGB color space is calculated from the estimated spectrum, illuminant spectrum and spectral responses of a display device.

\section{Experiments}

We made some experiments to evaluate the proposed estimation method. First, we measured the spectral reflectances of both the human skins and foundation materials in different conditions by using a spectrophotometer (Konika Minolta CM2600d). The skin spectral reflectances were measured without makeup and with foundation makeup. The surface reflectances of the foundation materials were measured in a thick layer and a thin layer on a black background. The subjects were five young women. The colors of foundations used are six colors of bright beige $(\mathrm{Bb})$, beige $(\mathrm{Bc})$, dark beige $(\mathrm{Bd})$, bright ocre $(\mathrm{Ob})$, ocre $(\mathrm{Oc})$, and dark ocre $(\mathrm{Od})$. The measured area was the inside of a circle with $8 \mathrm{~mm}$ diameters on the cheek of each subject. The wavelength range was from $400 \mathrm{~nm}$ to $700 \mathrm{~nm}$ with the interval of $10 \mathrm{~nm}$. The absorption coefficients and scattering coefficients of foundations were derived from the spectral reflectances for the thick and thin layers of foundations by Equations (4) and (5).

Next, the spectral reflectance of skin with foundation makeup was estimated. We determined the skin surface $R_{S}$ and the thickness of foundation layer so that the estimated spectral reflectance $R(\lambda)$ of Equation (6) can be fitted to the measured spectral reflectance in the sense of least squared error.

Figure 6 shows the estimation result for one subject with the foundation $\mathrm{Bb}$. It compares the estimated spectral curve of skin reflectance with the foundation makeup to the direct measurement the same skin reflectance. The figure demonstrates a good accuracy of our estimation results. Table 1 shows numerical errors between the estimated reflectance and the measurements. Note that the errors are quite small. Table 2 shows the average value of estimated thickness for each foundation over five subjects. 
The thickness values were almost same as the thickness that a person spreads foundation on her cheek with. Table 3 lists the average value of the estimated specular reflectance $R s$ for each foundation over five subjects. These values are convincing because the foundations we used had matt effect.

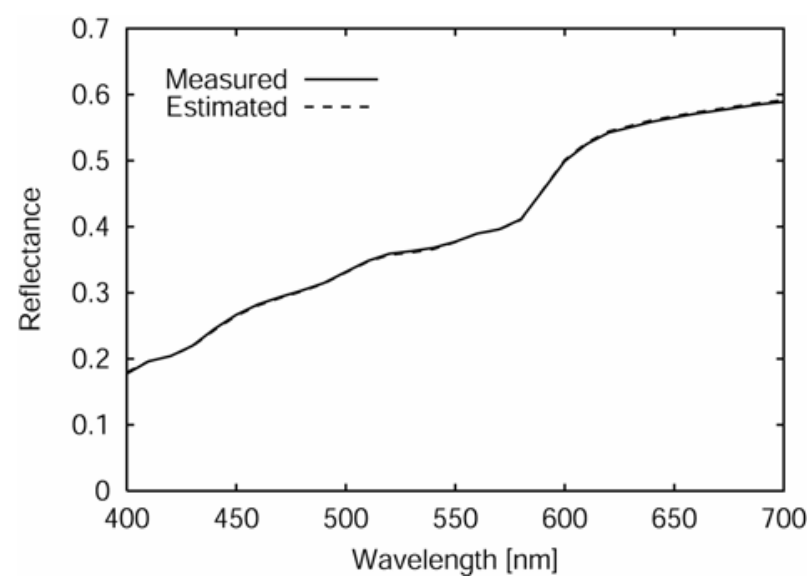

Fig. 6. A result of the estimation

Table 1. Errors in the estimation

\begin{tabular}{|c|c|c|c|c|c|c|c|}
\hline & $\mathrm{Bb}$ & $\mathrm{Bc}$ & $\mathrm{Bd}$ & $\mathrm{Ob}$ & $\mathrm{Oc}$ & Od & Average \\
\hline $\begin{array}{c}\text { Min } \\
\left(\times 10^{-6}\right) \\
\text { Max }\end{array}$ & 36 & 332 & 178 & 497 & 352 & 89 & - \\
$\left(\times 10^{-6}\right)$ & 1696 & 1360 & 1799 & 4539 & 2225 & 1793 & - \\
\hline $\begin{array}{c}\text { Ave } \\
\left(\times 10^{-6}\right)\end{array}$ & 885 & 732 & 848 & 2031 & 1102 & 1003 & 1100 \\
\hline
\end{tabular}

Table 2. Average values of the estimated thickness values over five subjects

\begin{tabular}{|l|c|c|c|c|c|c|}
\hline & $\mathrm{Bb}$ & $\mathrm{Bc}$ & $\mathrm{Bd}$ & $\mathrm{Ob}$ & $\mathrm{Oc}$ & $\mathrm{Od}$ \\
\hline Thickness $[\mu \mathrm{m}]$ & 1.8 & 2.5 & 2.8 & 2.8 & 3.4 & 4.1 \\
\hline
\end{tabular}

Table 3. Average values of estimated specular reflectance values over five subjects

\begin{tabular}{|l|c|c|c|c|c|c|}
\hline & $\mathrm{Bb}$ & $\mathrm{Bc}$ & $\mathrm{Bd}$ & $\mathrm{Ob}$ & $\mathrm{Oc}$ & Od \\
\hline $\begin{array}{l}\text { Specular reflec- } \\
\text { tance [\%] }\end{array}$ & 2.27 & 1.94 & 1.79 & 0.49 & 1.65 & 0.78 \\
\hline
\end{tabular}




\section{Application to a Skin Color Simulator}

We have developed a color simulator for human skin with foundation makeup. The simulator is programmed by Microsoft Visual Basic and has a graphical user interface (GUI). Figure 7 shows the windows of the GUI. A user selects the type of cosmetics in the window in Figure 7 (a). Next, the system estimates the spectral reflectance of skin with the foundation makeup. The skin color is then calculated in terms of the tristimulus values CIE-XYZ under such a standard illumination as CIE D65. Finally, the color values are transformed into the device RGB values to produce realistic skin colors on a calibrated CRT display. Figure 7 (b) shows the display window, where the color inside the center circle shows the original skin color without makeup. Thus the user can easily compare the color of her original bare skin without makeup to the skin with any foundation makeup.

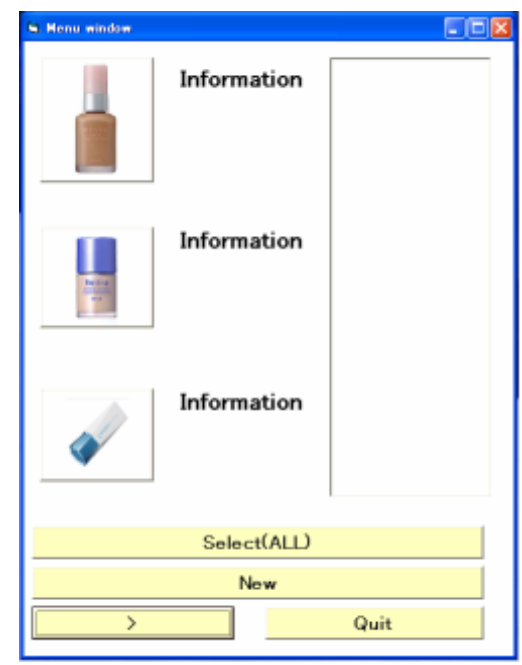

(a) Cosmetics selector window

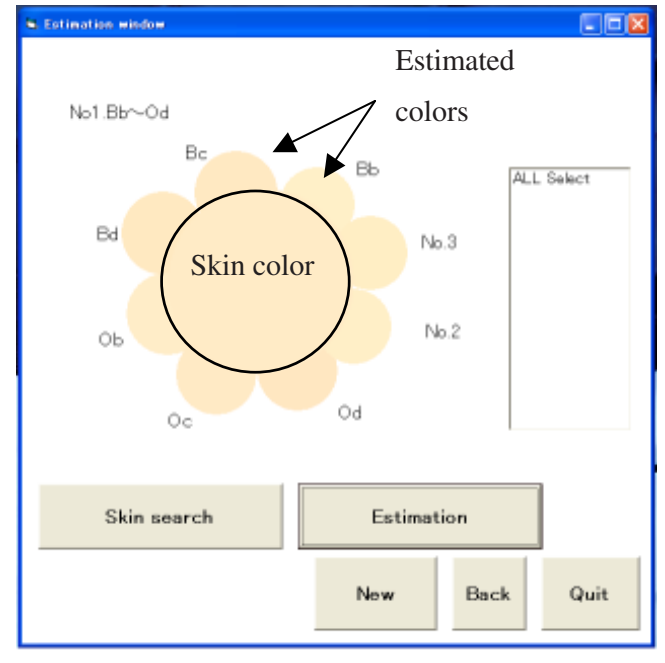

(b) Estimated color display window

Fig. 7. The GUI for the color simulation

\section{Conclusions}

In this paper, we described the optical model of skin with makeup, an estimation algorithm of surface-spectral reflectance and a color simulator using the estimated reflectance. The present paper modeled the optics of skin with foundation makeup by two layers. The surface spectrum of skin with foundation makeup was estimated by the Kubelka-Munk theory for radiation transfer in the turbid medium. The proposed algorithm can predict the spectral shape of skin surface with different types of foundation by appropriately determining model parameters. The object color of human skin with makeup was created from three kinds of spectral data of surface-spectral reflec- 
tance, an illuminant spectrum, and the spectral response of a display device used for display. As an application of the skin reflectance estimation, we have developped a color simulator for human skin with foundation makeup. Experimental results showed a good feasibility of the proposed method.

\section{Acknowledgments}

We appreciate Kanebo Cosmetics Inc. Cosmetic Laboratory supporting for measurement of skin reflectance with foundation makeup.

\section{References}

1. Angelopoulou E., Molana R., Danilidis K.: Multispectral skin color modeling, Proceedings of IEEE Conference of Computer Vision and Pattern Recognition (2001) 635-442

2. Doi M., Tanaka N., Tominaga S.: Spectral Reflectance-Based Modeling of Human Skin and its Application, Proceedings of 2nd European Conference on Color in Graphics, Imaging and Vision (2004) 388-392

3. Kubelka P.: New Contributions to the Optics of Intensely Light-Scattering Materials. Part I, Journal of Optical Society of America, vol. 38, no. 5. (1948) 448-457

4. Kubelka P.: New Contributions to the Optics of Intensely Light-Scattering Materials. Part II: Nonhomogeneous Layers, Journal of Optical Society of America, vol. 44, no. 4. (1954) 330-335

5. Tsumura N., Ojima N., Sato K., Shiraishi M., Shimizu H., Nabeshima H., Akazaki S., Hori K., Miyake Y.: Image-based skin color and texture analysis/synthesis by extracting hemoglobin and melanin information in the skin, acm Transactions on Graphics, vol. 22, no. 3. (2003) 770-779. 